\title{
Second-order optimality and duality in vector optimization over cones
}

\author{
S. K. Suneja ${ }^{1}$, Sunila Sharma ${ }^{2}$, Malti Kapoor ${ }^{3, *}$ \\ ${ }^{1}$ Department of Mathematics, University of Delhi, India. \\ ${ }^{2}$ Department of Mathematics, Miranda House, University of Delhi, India. \\ ${ }^{3}$ Department of Mathematics, Motilal Nehru College, University of Delhi, India.
}

(Received: 5 October 2015; Accepted: 30 January 2016)

\begin{abstract}
In this paper, we introduce the notion of a second-order cone- convex function involving second-order directional derivative. Also, second-order cone-pseudoconvex, second-order cone-quasiconvex and other related functions are defined. Second-order optimality and Mond-Weir type duality results are derived for a vector optimization problem over cones using the introduced classes of functions.
\end{abstract}

Keywords Vector optimization over cones, Second-order cone-convexity, Second-order optimality conditions, Secondorder duality

AMS 2010 subject classifications 90C26, 90C29, 90C46

DOI: $10.19139 /$ soic.v4i2.171

\section{Introduction}

Generalized convexity notions have always been a significant aid in the progress of optimization theory. In 1981 Hanson [6] generalized convexity to invexity. Kaul and Kaur [11] named differentiable invex functions as $\eta$-convex and further generalized them to $\eta$-pseudoconvex and $\eta$-quasiconvex functions. Craven [5] extended the concept of convex functions to cone-convex functions. Recently Ivanov [7, 8, 9] illuminated the following definition of a second-order directional derivative obtained by solving the Taylor expansion formula of a function with respect to the second-order term.

Definition $1.1([7,8,9])$

Let $S$ be a nonempty open subset of $\mathbf{R}^{n}$ and $f: S \rightarrow \mathbf{R}$ be a differentiable function. The second-order directional derivative $f^{\prime \prime}(x, d)$ of $f$ at the point $x \in S$ in the direction $d \in \mathbf{R}^{n}$ is defined as an element of $\mathbf{R}$ given by

$$
f^{\prime \prime}(x, d)=\lim _{s \rightarrow 0^{+}} \frac{2}{s^{2}}[f(x+s d)-f(x)-s \nabla f(x) d] .
$$

If $f^{\prime \prime}(x, d)$ exists and is finite, then $f$ is said to be second-order directionally differentiable at the point $x \in S$ in the direction $d \in \mathbf{R}^{n}$ and $f^{\prime \prime}(x, d)$ is called its second-order directional derivative. The function $f$ is said to be second-order directionally differentiable on $S$ if the derivative $f^{\prime \prime}(x, d)$ exists for each $x \in S$ and every direction $d \in \mathbf{R}^{n}$.

\footnotetext{
*Correspondence to: Malti Kapoor (Email: maltikapoor1@ gmail.com). Department of Mathematics, Motilal Nehru College, University of Delhi, Delhi-110021, India.
}

ISSN 2310-5070 (online) ISSN 2311-004X (print)

Copyright (C) 2016 International Academic Press 
Remark 1.2

If $f$ is twice differentiable in a neighbourhood of $x \in S$, then $f^{\prime \prime}(x, d)$ exists for every $d \in \mathbf{R}^{n}$ and $f^{\prime \prime}(x, d)=$ $d^{t} \nabla^{2} f(x) d$, where $\nabla^{2} f(x)$ is the Hessian matrix of $f$ at $x$.

Ivanov [8] used the above definition of second-order directional derivative to introduce a second-order invex function in the following manner:

Definition $1.3([8])$

The function $f$ is said to be second-order invex (or 2-invex) at $x \in S$ if there exist vector-valued functions $\eta, \xi: S \times S \rightarrow \mathbf{R}^{n}$ such that for all $y \in S$, the second-order directional derivative $f^{\prime \prime}(x, \xi(x, y))$ exists and

$$
f(y)-f(x) \geq \nabla f(x) \eta(y, x)+f^{\prime \prime}(x, \xi(y, x)) .
$$

If the above inequality holds for all $x, y \in S$, then $f$ is called second-order invex on $S$.

In this paper, we unify the notions of $\eta$-convex functions [11] and second-order invex functions [8] to define a new class of second-order cone- $(\eta, \xi)$-convex functions. We also define related classes of second-order conepseudoconvex and second-order cone-quasiconvex functions.

Second-order optimality conditions for vector optimization problems have been widely studied in the past, mainly due to their usefulness in sensitivity analysis of optimal solutions and convergence analysis of various algorithms. Several researchers, like Andreani [1], Ben-Tal [2], Burke [4], Kawasaki [12], have considered secondorder optimality conditions in terms of Hessians of the involved functions. However, various kinds of second-order directional derivatives have also been introduced to enable the development of second-order optimality conditions in the absence of second-order differentiability (see for example Ben-Tal, Zowe [3], Ivanov [7, 8, 9], Studniarski [15], Yang [16] and the references therein).

We employ the introduced classes of functions to obtain second-order necessary and sufficient Karush-KuhnTucker (KKT) type conditions for a vector optimization problem over cones in terms of the second-order directional derivatives of the functions involved. Furthermore, a second-order Mond-Weir type dual is associated to the considered problem and weak and strong duality results are established.

\section{Second-order cone-convexity and related concepts}

In this section, we introduce the following new classes of second-order cone- $(\eta, \xi)$-convex functions.

Let $S$ be a nonempty open subset of $\mathbf{R}^{n}$ and $f=\left(f_{1}, \ldots, f_{m}\right)^{t}: S \rightarrow \mathbf{R}^{m}$ be a differentiable vector-valued function.

Definition 2.1

The function $f$ is said to be second-order $K-(\eta, \xi)$-convex at $\bar{x} \in S$ on $S$ if there exist vector-valued functions $\eta, \xi: S \times S \rightarrow \mathbf{R}^{n}$ such that for all $x \in S$, the second-order directional derivative $f^{\prime \prime}(\bar{x}, \xi(x, \bar{x}))$ exists and

$$
f(x)-f(\bar{x})-\nabla f(\bar{x}) \eta(x, \bar{x})-f^{\prime \prime}(\bar{x}, \xi(x, \bar{x})) \in K,
$$

where $\nabla f(\bar{x})=\left(\nabla f_{1}(\bar{x}), \ldots, \nabla f_{m}(\bar{x})\right)^{t}$ and $f^{\prime \prime}(\bar{x}, \xi(x, \bar{x}))=\left(f_{1}^{\prime \prime}(\bar{x}, \xi(x, \bar{x})), \ldots, f_{m}^{\prime \prime}(\bar{x}, \xi(x, \bar{x}))^{t}\right.$.

Remark 2.2 (i) If $f$ is a scalar valued function and $K=\mathbf{R}_{+}$, the above definition reduces to second-order invexity introduced by Ivanov [8].

(ii) If $\xi \equiv 0$ then Definition 2.1 becomes the definition of $K$-invexity given by Yen and Sach [17].

(iii) If $f$ is a scalar valued function, $K=\mathbf{R}_{+}$and $\xi \equiv 0$ then Definition 2.1 becomes the definition of $\eta$-convexity given by Kaul and Kaur [11].

(iv) If $\xi \equiv 0$ and $\eta(x, y)=x-y, x, y \in S$, then the above definition reduces to the definition of cone-convex functions introduced in [5]. 
To justify the introduction of second-order $K-(\eta, \xi)$-convex functions, we give an example of a function which is second-order $K-(\eta, \xi)$-convex but not $K$-invex.

Example 2.3

Let $S=\mathbf{R}$ and $K=\{(x, y): x \geq 0, y \geq x\}$. Consider the following function $f: S \rightarrow \mathbf{R}^{2}, f(x)=\left(f_{1}(x), f_{2}(x)\right)$.

$$
f_{1}(x)=\left\{\begin{array}{ll}
x^{2}, & x \geq 0 \\
-\frac{15}{7} x^{2}, & x<0
\end{array} \quad f_{2}(x)= \begin{cases}x^{3}+x^{2}, & x \geq 0 \\
-\frac{1}{2} x^{2}, & x<0\end{cases}\right.
$$

The function $f$ is second-order $K-(\eta, \xi)$-convex at the point $\bar{x}=0$, where $\xi(x, \bar{x})=\frac{1}{\sqrt{2}}(x-\bar{x})$ and $\eta$ is an arbitrary function. However, $f$ is not $K$-invex. For instance, at $x=-\frac{1}{2}$,

$$
f(x)-f(\bar{x})-\nabla f(\bar{x}) \eta(x, \bar{x})=\left(-\frac{15}{28},-\frac{1}{8}\right) \notin K .
$$

\section{Definition 2.4}

The function $f$ is said to be second-order $K-(\eta, \xi)$-pseudoconvex at $\bar{x} \in S$ on $S$ if there exist vector-valued functions $\eta, \xi: S \times S \rightarrow \mathbf{R}^{n}$ such that for all $x \in S$, the second-order directional derivative $f^{\prime \prime}(\bar{x}, \xi(x, \bar{x}))$ exists and

$$
-\left\{\nabla f(\bar{x}) \eta(x, \bar{x})+f^{\prime \prime}(\bar{x}, \xi(x, \bar{x}))\right\} \notin \operatorname{int} K \Rightarrow-\{f(x)-f(\bar{x})\} \notin \operatorname{int} K .
$$

Equivalently,

$$
\{f(x)-f(\bar{x})\} \in-\operatorname{int} K \Rightarrow\left\{\nabla f(\bar{x}) \eta(x, \bar{x})+f^{\prime \prime}(\bar{x}, \xi(x, \bar{x}))\right\} \in-\operatorname{int} K .
$$

Remark 2.5 (i) If $f$ is twice differentiable and $\xi=\eta$, then the above definition becomes the definition of a $K$ second order pseudoinvex function with respect to $\eta$ at $\bar{x}$ for $p=\eta(x, \bar{x}) \in \mathbf{R}^{n}$ introduced by Mishra and Lai [14].

(ii) If $\xi \equiv 0$ then Definition 2.4 becomes the definition of $K$-pseudoinvexity introduced by Khurana [13].

(iii) If $f$ is a scalar valued function, $K=\mathbf{R}_{+}$and $\xi \equiv 0$ then Definition 2.4 reduces to the definition of $\eta$-pseudo convexity given by Kaul and Kaur [11].

It is clear that every second-order $K-(\eta, \xi)$-convex function is $K-(\eta, \xi)$-pseudoconvex. However the converse may not be true as shown by the following example.

Example 2.6

Let $S=\mathbf{R}$ and $K=\{(x, y): y \leq 0, y \leq x\}$. Consider the following function $f: S \rightarrow \mathbf{R}^{2}, f(x)=\left(f_{1}(x), f_{2}(x)\right)$.

$$
f_{1}(x)=\left\{\begin{array}{ll}
x^{3}-x^{2}, & x \geq 0 \\
x^{4}-4 x^{2}, & x<0
\end{array} \quad f_{2}(x)= \begin{cases}x^{3}+2 x^{2}, & x \geq 0 \\
x^{3}, & x<0\end{cases}\right.
$$

At $\bar{x}=0$, we have

$$
f(x)-f(\bar{x}) \in-\operatorname{int} K \Rightarrow x>0 \Rightarrow \nabla f(\bar{x}) \eta(x, \bar{x})+f^{\prime \prime}(\bar{x}, \xi(x, \bar{x})) \in-\operatorname{int} K,
$$

where $\eta(x, \bar{x})=\bar{x}-x^{2}$ and $\xi(x, \bar{x})=(x-\bar{x})^{2}$.

Thus, $f$ is $K-(\eta, \xi)$-pseudoconvex at $\bar{x}=0$. However, $f$ fails to be $K-(\eta, \xi)$-convex at $\bar{x}=0$. If we take $x=\frac{1}{2}$,

$$
f(x)-f(\bar{x})-\nabla f(\bar{x}) \eta(x, \bar{x})-f^{\prime \prime}(\bar{x}, \xi(x, \bar{x}))=\left(0, \frac{3}{8}\right) \notin K .
$$




\section{Remark 2.7}

In the above example, $f$ is a vector-valued function which is not twice differentiable. Hence, $f$ is not $K$-second order pseudoinvex as defined by Mishra and Lai [14]. Further, if $\xi \equiv 0$, then $f$ is not $K$-pseudoinvex in the sense of Khurana [13]. Therefore Definition 2.4 widens the field of applications of generalized convex functions.

\section{Definition 2.8}

The function $f$ is said to be second-order $K-(\eta, \xi)$-strictly pseudoconvex at $\bar{x} \in S$ on $S$ if there exist vector-valued functions $\eta, \xi: S \times S \rightarrow \mathbf{R}^{n}$ such that for all $x \in S, f^{\prime \prime}(\bar{x}, \xi(x, \bar{x}))$ exists and

$$
-\{f(x)-f(\bar{x})\} \in K \Rightarrow-\left\{\nabla f(\bar{x}) \eta(x, \bar{x})+f^{\prime \prime}(\bar{x}, \xi(x, \bar{x}))\right\} \in \operatorname{int} K .
$$

Definition 2.9

The function $f$ is said to be second-order $K-(\eta, \xi)$-quasiconvex at $\bar{x} \in S$ on $S$ if there exist vector-valued functions $\eta, \xi: S \times S \rightarrow \mathbf{R}^{n}$ such that for all $x \in S, f^{\prime \prime}(\bar{x}, \xi(x, \bar{x}))$ exists and

$$
\{f(x)-f(\bar{x})\} \notin \operatorname{int} K \Rightarrow-\left\{\nabla f(\bar{x}) \eta(x, \bar{x})+f^{\prime \prime}(\bar{x}, \xi(x, \bar{x}))\right\} \in K .
$$

If $f$ is second-order $K-(\eta, \xi)$-convex (pseudoconvex, strictly pseudoconvex, quasiconvex) at every $\bar{x} \in S$ on $S$ then $f$ is said to be second-order $K-(\eta, \xi)$-convex (pseudoconvex, strictly pseudoconvex, quasiconvex) on $S$.

We shall study the following vector optimization problem (VOP) over cones:

$$
\text { (VOP) } \begin{aligned}
& K \text {-minimize } f(x) \\
& \text { subject to }-g(x) \in Q,
\end{aligned}
$$

where $f: S \rightarrow \mathbf{R}^{m}$ and $g: S \rightarrow \mathbf{R}^{p}$ are differentiable functions and $K \subseteq \mathbf{R}^{m}, Q \subseteq \mathbf{R}^{p}$ are closed convex cones with nonempty interior.

Let $S_{0}=\{x \in S:-g(x) \in Q\}$ denote the set of feasible solutions to (VOP).

\section{Definition 2.10}

Let $K \subseteq \mathbf{R}^{m}$ be a closed convex pointed cone with nonempty interior and let int $K$ denote the interior of $K$. The positive dual cone $K^{*}$ and the strict positive dual cone $K^{s^{*}}$ of $K$, are respectively defined as

$$
\begin{aligned}
& K^{*}=\left\{y^{*} \in \mathbf{R}^{m}:\left\langle y, y^{*}\right\rangle \geq 0 \text { for all } y \in K\right\}, \text { and } \\
& K^{s *}=\left\{y^{*} \in \mathbf{R}^{m}:\left\langle y, y^{*}\right\rangle>0 \text { for all } y \in K \backslash\{0\}\right\} .
\end{aligned}
$$

Definition 2.11

A point $\bar{x} \in S_{0}$ is said to be

(i) a weak minimum of (VOP) if for every $x \in S_{0}$,

$$
f(x)-f(\bar{x}) \notin-\operatorname{int} K \text {. }
$$

(ii) a minimum of (VOP) if for every $x \in S_{0}$,

$$
f(x)-f(\bar{x}) \notin-K \backslash\{0\} .
$$

\section{Second-order necessary conditions over cones}

We now prove second-order necessary optimality conditions for the problem (VOP) in terms of second-order directional derivatives.

Theorem 3.1

Let $\bar{x}$ be a weak minimum of (VOP). If $f^{\prime \prime}(\bar{x}, \cdot)$ is $K$-subconvexlike and $g^{\prime \prime}(\bar{x}, \cdot)$ is $Q$-subconvexlike on $\mathbf{R}^{n}$, then there exist $\lambda \in K^{*}, \mu \in Q^{*}$ not both zero, such that

$$
\begin{aligned}
& \lambda^{t}\left(\nabla f(\bar{x}) d_{1}+f^{\prime \prime}\left(\bar{x}, d_{2}\right)\right)+\mu^{t}\left(\nabla g(\bar{x}) d_{1}+g^{\prime \prime}\left(\bar{x}, d_{2}\right)\right) \geq 0, \quad \forall\left(d_{1}, d_{2}\right) \in \mathbf{R}^{n} \times \mathbf{R}^{n} \\
& \mu^{t} g(\bar{x})=0 .
\end{aligned}
$$


Proof

Let $G: \mathbf{R}^{n} \times \mathbf{R}^{n} \rightarrow \mathbf{R}^{m} \times \mathbf{R}^{p}$ be defined as

$$
G\left(d_{1}, d_{2}\right)=\left(\nabla f(\bar{x}) d_{1}+f^{\prime \prime}\left(\bar{x}, d_{2}\right), \nabla g(\bar{x}) d_{1}+g^{\prime \prime}\left(\bar{x}, d_{2}\right)+g(\bar{x})\right) .
$$

We assert that the system

$$
-G\left(d_{1}, d_{2}\right) \in \operatorname{int}(K \times Q),
$$

has no solution $\left(d_{1}, d_{2}\right) \in \mathbf{R}^{n} \times \mathbf{R}^{n}$.

If possible, let there be a solution $\left(\hat{d}_{1}, \hat{d}_{2}\right) \in \mathbf{R}^{n} \times \mathbf{R}^{n}$ of (3). Then,

$$
-G\left(\hat{d}_{1}, \hat{d}_{2}\right) \in \operatorname{int}(K \times Q) .
$$

That is, $-\left\{\nabla f(\bar{x}) \hat{d}_{1}+f^{\prime \prime}\left(\bar{x}, \hat{d}_{2}\right)\right\} \in \operatorname{int} K$,

and $-\left\{\nabla g(\bar{x}) \hat{d}_{1}+g^{\prime \prime}\left(\bar{x}, \hat{d}_{2}\right)+g(\bar{x})\right\} \in \operatorname{int} Q$.

Equivalently, $-\left[\nabla f(\bar{x}) \hat{d}_{1}+\lim _{s \rightarrow 0^{+}} \frac{2}{s^{2}}\left\{f\left(\bar{x}+s \hat{d}_{2}\right)-f(\bar{x})-s \nabla f(\bar{x}) \hat{d}_{2}\right\}\right] \in \operatorname{int} K$,

and $-\left[\nabla g(\bar{x}) \hat{d}_{1}+\lim _{s \rightarrow 0^{+}} \frac{2}{s^{2}}\left\{g\left(\bar{x}+s \hat{d}_{2}\right)-g(\bar{x})-s \nabla g(\bar{x}) \hat{d}_{2}\right\}+g(\bar{x})\right] \in \operatorname{int} Q$.

Or, $-\left\{f\left(\bar{x}+s \hat{d}_{2}\right)-f(\bar{x})\right\}+o(s) \in \operatorname{int} K$,

and $-g\left(\bar{x}+s \hat{d}_{2}\right)+o(s) \in-g(\bar{x})+\operatorname{int} Q$,

where $\lim _{s \rightarrow 0^{+}} o(s)=0$.

Since $S$ is a nonempty open set, we can find $s_{0}>0$ such that for all $s \in\left(0, s_{0}\right)$,

$$
\begin{aligned}
& \bar{x}+s \hat{d}_{2} \in S, \\
& -\left\{f\left(\bar{x}+s \hat{d}_{2}\right)-f(\bar{x})\right\} \in \operatorname{int} K,
\end{aligned}
$$

and $-g\left(\bar{x}+s \hat{d}_{2}\right) \in \operatorname{int} Q$,

which is a contradiction as $\bar{x}$ is a weak minimum of (VOP). Hence the system (3) has no solution $\left(d_{1}, d_{2}\right) \in$ $\mathbf{R}^{n} \times \mathbf{R}^{n}$. Also $G$ is $K \times Q$-subconvexlike on $\mathbf{R}^{n} \times \mathbf{R}^{n}$ as $f^{\prime \prime}(\bar{x}, \cdot)$ is $K$-subconvexlike and $g^{\prime \prime}(\bar{x}, \cdot)$ is $Q$ subconvexlike on $\mathbf{R}^{n}$.

Therefore by the Alternative Theorem given in Jeyakumar [10], there exist $\lambda \in K^{*}, \mu \in Q^{*}$ not both zero such that

$$
\lambda^{t}\left(\nabla f(\bar{x}) d_{1}+f^{\prime \prime}\left(\bar{x}, d_{2}\right)\right)+\mu^{t}\left(\nabla g(\bar{x}) d_{1}+g^{\prime \prime}\left(\bar{x}, d_{2}\right)+g(\bar{x})\right) \geq 0, \quad \forall\left(d_{1}, d_{2}\right) \in \mathbf{R}^{n} \times \mathbf{R}^{n} .
$$

Taking $d_{1}=d_{2}=0$ in the above inequality, we get $\mu^{t} g(\bar{x}) \geq 0$. Also, $\mu \in Q^{*},-g(\bar{x}) \in Q$ implies $\mu^{t} g(\bar{x}) \leq 0$. Thus, $\mu^{t} g(\bar{x})=0$.

Hence, from (4) we obtain

$$
\lambda^{t}\left(\nabla f(\bar{x}) d_{1}+f^{\prime \prime}\left(\bar{x}, d_{2}\right)\right)+\mu^{t}\left(\nabla g(\bar{x}) d_{1}+g^{\prime \prime}\left(\bar{x}, d_{2}\right)\right) \geq 0, \quad \forall\left(d_{1}, d_{2}\right) \in \mathbf{R}^{n} \times \mathbf{R}^{n} .
$$

Now we give an example to illustrate the result obtained in Theorem 3.1.

Example 3.2

Let $S=\mathbf{R}^{2}$. Consider the functions $f: S \rightarrow \mathbf{R}^{2}, f(x)=\left(f_{1}(x), f_{2}(x)\right)$ and $g: S \rightarrow \mathbf{R}^{2}, g(x)=\left(g_{1}(x), g_{2}(x)\right)$.

$$
\begin{aligned}
& f_{1}(x)=\left\{\begin{array}{ll}
-x_{1}^{2}-1, & x_{1} \geq 0 \\
-2 x_{2}^{2}-1, & x_{1}<0
\end{array} \quad f_{2}(x)= \begin{cases}x_{1}^{2}+x_{2}^{4}+1, & x_{1} \geq 0 \\
2 x_{2}^{2}+1, & x_{1}<0\end{cases} \right. \\
& g_{1}(x)=\left\{\begin{array}{ll}
x_{2}-x_{1}^{2}-x_{1}^{3}, & x_{1} \geq 0 \\
x_{2}-\frac{1}{2} x_{1}^{2}, & x_{1}<0
\end{array} \quad g_{2}(x)= \begin{cases}x_{2}^{2}+x_{2}^{3}, & x_{1} \geq 0 \\
4 x_{2}^{2}, & x_{1}<0\end{cases} \right.
\end{aligned}
$$


Clearly $\bar{x}=(0,0)$ is a weak minimum for the problem,

(VOP) $\quad K$-minimize $f(x)$

subject to $-g(x) \in Q$,

where $K=\left\{\left(x_{1}, x_{2}\right): x_{1} \geq 0, x_{2} \geq x_{1}\right\}$ and $Q=\left\{\left(x_{1}, x_{2}\right): x_{1} \leq 0, x_{2} \geq 0\right\}$.

We have, for $d=(\alpha, \beta) \in \mathbf{R}^{2}$,

$$
f^{\prime \prime}(\bar{x}, d)= \begin{cases}\left(-2 \alpha^{2}, 2 \alpha^{2}\right), & \alpha \geq 0 \\ \left(-4 \beta^{2}, 4 \beta^{2}\right), & \alpha<0\end{cases}
$$

and

$$
g^{\prime \prime}(\bar{x}, d)= \begin{cases}\left(-2 \alpha^{2}, 2 \beta^{2}\right), & \alpha \geq 0 \\ \left(-\alpha^{2}, 8 \beta^{2}\right), & \alpha<0\end{cases}
$$

It can be seen that $f^{\prime \prime}(\bar{x}, \cdot)$ is $K$-subconvexlike and $g^{\prime \prime}(\bar{x}, \cdot)$ is $Q$-subconvexlike on $\mathbf{R}^{2}$.

If we take $\lambda=\left(-\frac{1}{4}, \frac{1}{2}\right)$ and $\mu=\left(0, \frac{7}{8}\right)$, then for all $\left(d_{1}, d_{2}\right) \in \mathbf{R}^{2} \times \mathbf{R}^{2}$, we have

$$
\begin{aligned}
& \lambda^{t}\left(\nabla f(\bar{x}) d_{1}+f^{\prime \prime}\left(\bar{x}, d_{2}\right)\right)+\mu^{t}\left(\nabla g(\bar{x}) d_{1}+g^{\prime \prime}\left(\bar{x}, d_{2}\right)\right) \geq 0, \\
& \mu^{t} g(\bar{x})=0 .
\end{aligned}
$$

We now introduce the following second-order Slater-type constraint qualification over cones.

\section{Definition 3.3}

The problem (VOP) is said to satisfy second-order Slater-type cone-constraint qualification at $\bar{x}$ if $g$ is $Q-(\eta, \xi)$ convex at $\bar{x}$ and there exists $\hat{x} \in S$ such that $-g(\hat{x}) \in \operatorname{int} Q$.

\section{Theorem 3.4}

Let $\bar{x}$ be a weak minimum of (VOP) at which second-order Slater-type cone-constraint qualification holds. If $f^{\prime \prime}(\bar{x}, \cdot)$ is $K$-subconvexlike and $g^{\prime \prime}(\bar{x}, \cdot)$ is $Q$-subconvexlike on $\mathbf{R}^{n}$, then there exist $\lambda \in K^{*} \backslash\{0\}$ and $\mu \in Q^{*}$ such that (1) and (2) hold.

Proof

Since $\bar{x}$ is a weak minimum of (VOP), by Theorem 3.1, there exist $\lambda \in K^{*}, \mu \in Q^{*}$, not both zero, such that (1) and (2) hold.

Since second-order Slater-type cone-constraint qualification holds at $\bar{x}, g$ is $Q-(\eta, \xi)$-convex at $\bar{x}$ and there exists $\hat{x} \in S$ such that $-g(\hat{x}) \in \operatorname{int} Q$. We have to prove that $\lambda \neq 0$.

Let if possible $\lambda=0$, then $\mu \neq 0$ and from (1) we get

$$
\mu^{t}\left(\nabla g(\bar{x}) d_{1}+g^{\prime \prime}\left(\bar{x}, d_{2}\right)\right) \geq 0, \quad \forall\left(d_{1}, d_{2}\right) \in \mathbf{R}^{n} \times \mathbf{R}^{n} .
$$

Also since $g$ is $Q-(\eta, \xi)$-convex at $\bar{x}$ there exist vector-valued functions $\eta, \xi: S \times S \rightarrow \mathbf{R}^{n}$ such that for all $x \in S$, the second-order directional derivative $g^{\prime \prime}(\bar{x}, \xi(x, \bar{x}))$ exists and

$$
g(x)-g(\bar{x})-\nabla g(\bar{x}) \eta(x, \bar{x})-g^{\prime \prime}(\bar{x}, \xi(x, \bar{x})) \in Q,
$$

which gives, $\mu^{t}\left\{g(x)-g(\bar{x})-\nabla g(\bar{x}) \eta(x, \bar{x})-g^{\prime \prime}(\bar{x}, \xi(x, \bar{x}))\right\} \geq 0$.

Using (2) and (5) for $d_{1}=\eta(x, \bar{x}), d_{2}=\xi(x, \bar{x})$ in the above inequality, we get $\mu^{t} g(x) \geq 0, \forall x \in S$, which contradicts $-g(\hat{x}) \in$ int $Q, \mu \in Q^{*}, \mu \neq 0$ implying $\mu^{t} g(\hat{x})<0$. Hence, $\lambda \neq 0$.

Theorem 3.5

Let $\bar{x}$ be a weak minimum of (VOP) at which second-order Slater-type cone-constraint qualification holds. If $f^{\prime \prime}(\bar{x}, \cdot)$ is $K$-subconvexlike and $g^{\prime \prime}(\bar{x}, \cdot)$ is $Q$-subconvexlike on $\mathbf{R}^{n}$, then there exist $\lambda \in K^{*} \backslash\{0\}$ and $\mu \in Q^{*}$ such that

$$
\begin{aligned}
& \lambda^{t} \nabla f(\bar{x})+\mu^{t} \nabla g(\bar{x})=0, \\
& \lambda^{t} f^{\prime \prime}(\bar{x}, d)+\mu^{t} g^{\prime \prime}(\bar{x}, d) \geq 0, \quad \forall d \in \mathbf{R}^{n} \\
& \mu^{t} g(\bar{x})=0 .
\end{aligned}
$$


Proof

Since all the conditions of Theorem 3.4 hold, there exist $\lambda \in K^{*} \backslash\{0\}$ and $\mu \in Q^{*}$ such that (1) holds along with $\mu^{t} g(\bar{x})=0$.

Taking $d_{2}=0$ in (1), we have

$$
\lambda^{t} \nabla f(\bar{x}) d_{1}+\mu^{t} \nabla g(\bar{x}) d_{1} \geq 0, \quad \forall d_{1} \in \mathbf{R}^{n} .
$$

As the above inequality holds for all $d_{1} \in \mathbf{R}^{n}$, we get

$$
\lambda^{t} \nabla f(\bar{x})+\mu^{t} \nabla g(\bar{x})=0 .
$$

Again, taking $d_{1}=0$ in (1), we obtain

$$
\begin{array}{ll} 
& \lambda^{t} f^{\prime \prime}\left(\bar{x}, d_{2}\right)+\mu^{t} g^{\prime \prime}\left(\bar{x}, d_{2}\right) \geq 0, \quad \forall d_{2} \in \mathbf{R}^{n} . \\
\text { Or, } \quad & \lambda^{t} f^{\prime \prime}(\bar{x}, d)+\mu^{t} g^{\prime \prime}(\bar{x}, d) \geq 0, \quad \forall d \in \mathbf{R}^{n} .
\end{array}
$$

This completes the proof.

\section{Second-order sufficient optimality conditions over cones}

We now provide several second-order sufficient conditions for the existence of a weak minimum or minimum for (VOP).

\section{Theorem 4.1}

Let $f$ be second-order $K-(\eta, \xi)$-convex and g be second-order $Q-(\eta, \xi)$-convex at $\bar{x} \in S_{0}$ on $S$, where $\eta, \xi: S \times S \rightarrow \mathbf{R}^{n}$. Suppose that there exist $\bar{\lambda} \in K^{*} \backslash\{0\}$ and $\bar{\mu} \in Q^{*}$ such that

$$
\begin{aligned}
& \bar{\lambda}^{t}\left(\nabla f(\bar{x}) d_{1}+f^{\prime \prime}\left(\bar{x}, d_{2}\right)\right)+\bar{\mu}^{t}\left(\nabla g(\bar{x}) d_{1}+g^{\prime \prime}\left(\bar{x}, d_{2}\right)\right) \geq 0, \quad \forall d_{1}, d_{2} \in \mathbf{R}^{n} \\
& \bar{\mu}^{t} g(\bar{x})=0,
\end{aligned}
$$

then $\bar{x}$ is a weak minimum of (VOP).

Proof

Assume on the contrary that $\bar{x}$ is not a weak minimum of (VOP), then there exists $\hat{x} \in S_{0}$ such that

$$
f(\bar{x})-f(\hat{x}) \in \operatorname{int} K \text {. }
$$

Since $f$ is $K-(\eta, \xi)$-convex at $\bar{x}$ on $S$, we have

$$
f(\hat{x})-f(\bar{x})-\nabla f(\bar{x}) \eta(\hat{x}, \bar{x})-f^{\prime \prime}(\bar{x}, \xi(\hat{x}, \bar{x})) \in K .
$$

Adding (11) and (12), we get $-\nabla f(\bar{x}) \eta(\hat{x}, \bar{x})-f^{\prime \prime}(\bar{x}, \xi(\hat{x}, \bar{x})) \in \operatorname{int} K$.

As, $\bar{\lambda} \in K^{*} \backslash\{0\}$, we obtain

$$
\bar{\lambda}^{t} \nabla f(\bar{x}) \eta(\hat{x}, \bar{x})+\bar{\lambda}^{t} f^{\prime \prime}(\bar{x}, \xi(\hat{x}, \bar{x}))<0 .
$$

Similarly, $g$ is $Q-(\eta, \xi)$-convex at $\bar{x}$ on $S$, therefore $g(\hat{x})-g(\bar{x})-\nabla g(\bar{x}) \eta(\hat{x}, \bar{x})-g^{\prime \prime}(\bar{x}, \xi(\hat{x}, \bar{x})) \in Q$, so that, $\bar{\mu}^{t}\left\{g(\hat{x})-g(\bar{x})-\nabla g(\bar{x}) \eta(\hat{x}, \bar{x})-g^{\prime \prime}(\bar{x}, \xi(\hat{x}, \bar{x}))\right\} \geq 0$.

However, $\hat{x} \in S_{0}$ and $\bar{\mu} \in Q^{*}$ implies $\bar{\mu}^{t} g(\hat{x}) \leq 0$. This together with (10) gives

$$
\bar{\mu}^{t} \nabla g(\bar{x}) \eta(\hat{x}, \bar{x})+\bar{\mu}^{t} g^{\prime \prime}(\bar{x}, \xi(\hat{x}, \bar{x})) \leq 0 .
$$

Adding (13) and (14), we get

$$
\bar{\lambda}^{t}\left(\nabla f(\bar{x}) \eta(\hat{x}, \bar{x})+f^{\prime \prime}(\bar{x}, \xi(\hat{x}, \bar{x}))\right)+\bar{\mu}^{t}\left(\nabla g(\bar{x}) \eta(\hat{x}, \bar{x})+g^{\prime \prime}(\bar{x}, \xi(\hat{x}, \bar{x}))\right)<0,
$$

which is a contradiction to (9) for $d_{1}=\eta(\hat{x}, \bar{x}), d_{2}=\xi(\hat{x}, \bar{x})$. 


\section{Theorem 4.2}

Let $f$ be second-order $K-(\eta, \xi)$-pseudoconvex and $g$ be second-order $Q-(\eta, \xi)$-quasiconvex at $\bar{x} \in S_{0}$ on $S$, where $\eta, \xi: S \times S \rightarrow \mathbf{R}^{n}$. Assume that there exist $\bar{\lambda} \in K^{*} \backslash\{0\}$ and $\bar{\mu} \in Q^{*}$ such that (9) and (10) hold. Then $\bar{x}$ is a weak minimum of (VOP).

\section{Proof}

Suppose to the contrary that $\bar{x}$ is not a weak minimum of (VOP), then there exists $\hat{x} \in S_{0}$ such that $f(\bar{x})-f(\hat{x}) \in$ int $K$.

By second-order $K$ - $(\eta, \xi)$-pseudoconvexity of $f$ at $\bar{x}$, we get $\nabla f(\bar{x}) \eta(\hat{x}, \bar{x})+f^{\prime \prime}(\bar{x}, \xi(\hat{x}, \bar{x})) \in-\operatorname{int} K$.

Since $\bar{\lambda} \in K^{*} \backslash\{0\}$, we have

$$
\bar{\lambda}^{t} \nabla f(\bar{x}) \eta(\hat{x}, \bar{x})+\bar{\lambda}^{t} f^{\prime \prime}(\bar{x}, \xi(\hat{x}, \bar{x}))<0 .
$$

Also, $\hat{x} \in S_{0}$ implies $\bar{\mu}^{t} g(\hat{x}) \leq 0$. On using (10), we get $\bar{\mu}^{t}\{g(\hat{x})-g(\bar{x})\} \leq 0$.

If $\bar{\mu} \neq 0$, then we have $g(\hat{x})-g(\bar{x}) \notin \operatorname{int} Q$.

Again, by second-order $Q-(\eta, \xi)$-quasiconvexity of $g$ at $\bar{x}$, we obtain

$$
\nabla g(\bar{x}) \eta(\hat{x}, \bar{x})+g^{\prime \prime}(\bar{x}, \xi(\hat{x}, \bar{x})) \in-Q .
$$

Therefore,

$$
\bar{\mu}^{t} \nabla g(\bar{x}) \eta(\hat{x}, \bar{x})+\bar{\mu}^{t} g^{\prime \prime}(\bar{x}, \xi(\hat{x}, \bar{x})) \leq 0 .
$$

If $\bar{\mu}=0$, then also (16) holds.

Now proceeding as in last part of Theorem 4.1, we arrive at a contradiction.

Hence, $\bar{x}$ is a weak minimum of (VOP).

\section{Theorem 4.3}

Let $f$ be second-order $K-(\eta, \xi)$-convex and $g$ be second-order $Q$ - $(\eta, \xi)$-convex at $\bar{x} \in S_{0}$ on $S$, where $\eta, \xi$ : $S \times S \rightarrow \mathbf{R}^{n}$. Suppose that there exist $\bar{\lambda} \in K^{s^{*}}$ and $\bar{\mu} \in Q^{*}$ such that (9) and (10) hold. Then $\bar{x}$ is a minimum of (VOP).

Proof

Let if possible, $\bar{x}$ be not a minimum of (VOP), then there exists $\hat{x} \in S_{0}$ such that

$$
f(\bar{x})-f(\hat{x}) \in K \backslash\{0\} .
$$

As $f$ is second-order $K-(\eta, \xi)$-convex at $\bar{x}$ on $S$, we have

$$
f(\hat{x})-f(\bar{x})-\nabla f(\bar{x}) \eta(\hat{x}, \bar{x})-f^{\prime \prime}(\bar{x}, \xi(\hat{x}, \bar{x})) \in K .
$$

From (17) and (18), we get

$$
-\left[\nabla f(\bar{x}) \eta(\hat{x}, \bar{x})+f^{\prime \prime}(\bar{x}, \xi(\hat{x}, \bar{x}))\right] \in K \backslash\{0\} .
$$

Since $\bar{\lambda} \in K^{s^{*}}$, we obtain

$$
\bar{\lambda}^{t} \nabla f(\bar{x}) \eta(\hat{x}, \bar{x})+\bar{\lambda}^{t} f^{\prime \prime}(\bar{x}, \xi(\hat{x}, \bar{x}))<0 .
$$

This leads to a contradiction as in Theorem 4.1. Hence $\bar{x}$ is a minimum of (VOP).

Theorem 4.4

Let $f$ be second-order $K-(\eta, \xi)$-strictly pseudoconvex ant $g$ be second-order $Q$-( $\eta, \xi)$-quasiconvex at $\bar{x} \in S_{0}$ on $S$, where $\eta, \xi: S \times S \rightarrow \mathbf{R}^{n}$. Assume that there exist $\bar{\lambda} \in K^{*} \backslash\{0\}$ and $\bar{\mu} \in Q^{*}$ such that (9) and (10) hold. Then $\bar{x}$ is a minimum of (VOP). 
Proof

Suppose to the contrary that $\bar{x}$ is not a minimum of (VOP). Then there exists $\hat{x} \in S_{0}$ such that (17) holds.

Since $f$ is second-order $K-(\eta, \xi)$-strictly pseudoconvex at $\bar{x}$ on $S$, therefore, we have

$$
-\left[\nabla f(\bar{x}) \eta(\hat{x}, \bar{x})+f^{\prime \prime}(\bar{x}, \xi(\hat{x}, \bar{x}))\right] \in \operatorname{int} K .
$$

Proceeding on the lines of Theorem 4.2, we obtain a contradiction. Hence $\bar{x}$ is a minimum of (VOP).

\section{Second-order Mond-Weir type duality over cones}

We associate the following Mond-Weir type dual problem (VUD) with the primal problem (VOP):

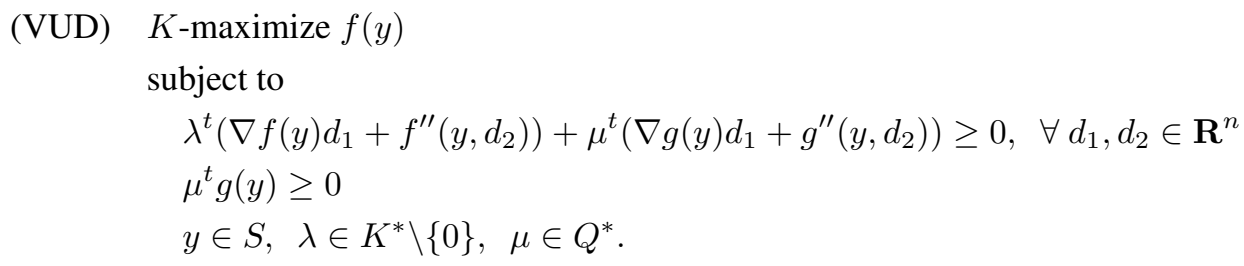

Let $S_{D}$ denote the set of feasible solutions of (VUD).

Theorem 5.1 (Weak Duality)

Let $x \in S_{0}$ and $(y, \lambda, \mu) \in S_{D}$. Assume that $f$ is second-order $K-(\eta, \xi)$-convex and $g$ is second-order $Q-(\eta, \xi)$ convex at $y$ on $S$, where $\eta, \xi: S \times S \rightarrow \mathbf{R}^{n}$. Then,

$$
f(y)-f(x) \notin \operatorname{int} K \text {. }
$$

Proof

Let if possible,

$$
f(y)-f(x) \in \operatorname{int} K
$$

Since $f$ is second-order $K-(\eta, \xi)$-convex at $y$ on $S$, we have

$$
f(x)-f(y)-\nabla f(y) \eta(x, y)-f^{\prime \prime}(y, \xi(x, y)) \in K .
$$

Adding (22) and (23), we get $-\nabla f(y) \eta(x, y)-f^{\prime \prime}(y, \xi(x, y)) \in \operatorname{int} K$.

As, $\lambda \in K^{*} \backslash\{0\}$, we obtain

$$
\lambda^{t} \nabla f(y) \eta(x, y)+\lambda^{t} f^{\prime \prime}(y, \xi(x, y))<0 .
$$

Also, $\mu \in Q^{*}$ and $g$ is second-order $Q-(\eta, \xi)$-convex at $y$ on $S$, therefore

$$
\mu^{t}\left[g(x)-g(y)-\nabla g(y) \eta(x, y)-g^{\prime \prime}(y, \xi(x, y))\right] \geq 0 .
$$

Adding (24) and (25), we have

$$
\mu^{t} g(x)-\mu^{t} g(y)>\lambda^{t}\left[\nabla f(y) \eta(x, y)+f^{\prime \prime}(y, \xi(x, y))\right]+\mu^{t}\left[\nabla g(y) \eta(x, y)+g^{\prime \prime}(y, \xi(x, y))\right] .
$$

Since $(y, \lambda, \mu) \in S_{D}$, therefore (20) holds for $d_{1}=\eta(x, y), d_{2}=\xi(x, y)$ and we get $\mu^{t} g(y)<\mu^{t} g(x)$. As $x \in S_{0}$ and $\mu \in Q^{*}$, we have $\mu^{t} g(x) \leq 0$, so that $\mu^{t} g(y)<0$. This contradicts the feasibility of $(y, \lambda, \mu)$ and completes the proof. 
Theorem 5.2 (Weak Duality)

Let $x \in S_{0}$ and $(y, \lambda, \mu) \in S_{D}$. Suppose $f$ is second-order $K-(\eta, \xi)$-pseudoconvex and $g$ is second-order $Q$ - $(\eta, \xi)$ quasiconvex at $y$ on $S$, where $\eta, \xi: S \times S \rightarrow \mathbf{R}^{n}$. Then,

$$
f(y)-f(x) \notin \operatorname{int} K \text {. }
$$

Proof

The proof follows on the lines of Theorem 4.2.

Theorem 5.3 (Strong Duality)

Suppose $\bar{x}$ is a weak minimum of (VOP) and second-order Slater-type cone-constraint qualification holds at $\bar{x}$. Further assume that $f^{\prime \prime}(\bar{x}, \cdot)$ is $K$-subconvexlike and $g^{\prime \prime}(\bar{x}, \cdot)$ is $Q$-subconvexlike on $\mathbf{R}^{n}$, then there exist $\bar{\lambda} \in K^{*} \backslash\{0\}, \bar{\mu} \in Q^{*}$ such that $(\bar{x}, \bar{\lambda}, \bar{\mu})$ is a feasible solution of (VUD). Moreover, if the conditions of Weak Duality Theorem 5.1 (Theorem 5.2) hold for all $x \in S_{0}$ and $(y, \lambda, \mu) \in S_{D}$, then $(\bar{x}, \bar{\lambda}, \bar{\mu})$ is a weak maximum of (VUD) and the objective function values for (VOP) and (VUD) are equal.

Proof

Since all the conditions of Theorem 3.4 are satisfied, therefore there exist $\bar{\lambda} \in K^{*} \backslash\{0\}, \bar{\mu} \in Q^{*}$ such that

$$
\begin{aligned}
& \bar{\lambda}^{t}\left(\nabla f(\bar{x}) d_{1}+f^{\prime \prime}\left(\bar{x}, d_{2}\right)\right)+\bar{\mu}^{t}\left(\nabla g(\bar{x}) d_{1}+g^{\prime \prime}\left(\bar{x}, d_{2}\right)\right) \geq 0, \quad \forall d_{1}, d_{2} \in \mathbf{R}^{n}, \\
& \bar{\mu}^{t} g(\bar{x})=0 .
\end{aligned}
$$

Thus, $(\bar{x}, \bar{\lambda}, \bar{\mu}) \in S_{D}$ and the objective function values for (VOP) and (VUD) are equal. Further if $(\bar{x}, \bar{\lambda}, \bar{\mu})$ is not a weak maximum of (VUD), then there exists $(y, \lambda, \mu) \in S_{D}$ such that

$$
f(y)-f(\bar{x}) \in \operatorname{int} K,
$$

which contradicts Theorem 5.1 (Theorem 5.2). Hence $(\bar{x}, \bar{\lambda}, \bar{\mu})$ is a weak maximum of (VUD).

\section{Conclusions}

In this paper, we use the idea of cones to generalize the definition of second-order invex functions given by Ivanov [8]. The second-order optimality conditions obtained herein apply to a wider range of functions than others in the literature. The fundamental contribution of the paper is the second-order necessary optimality conditions which aid the search for optimal solutions of (VOP) by reducing the candidate set of solutions in the absence of second-order differentiability.

\section{REFERENCES}

1. R. Andreani, R. Behling, G. Haeser and P.J.S. Silva, On second-order optimality conditions for nonlinear optimization, To appear (available online), 2014.

2. A. Ben-Tal, Second-order and related extremality conditions in nonlinear programming, J. Optim. Theory Appl., vol. 31, no. 2, pp. $143-165,1980$.

3. A. Ben-Tal and J. Zowe, Necessary and sufficient optimality conditions for a class of nonsmooth minimization problems, Math. Program., vol. 24, pp. 70-91, 1982.

4. J.V. Burke, Second order necessary and sufficient conditions for convex composite NDO, Math. Program., vol. 38, pp. 287-302, 1987.

5. B.D. Craven, Lagrangian conditions and quasiduality, Bull. of Australian Math. Soc., vol. 9, pp. 181-192, 1987.

6. M.A. Hanson, On sufficiency of the Kuhn-Tucker conditions, J. Math. Anal. Appl., vol. 80, pp. 545-550, 1981.

7. V.I. Ivanov, Second-order Kuhn-Tucker invex constrained problems, J. Glob. Optim., vol. 50, pp. 519-529, 2011.

8. V.I. Ivanov, Second-order invex functions in nonlinear programming, Optimization, vol. 61, no. 5, pp. 489-503, 2012.

9. V.I. Ivanov, Duality in nonlinear programming, Optim. Lett., vol. 7, no. 8, pp. 1643-1658, 2013.

10. V. Jeyakumar, Convexlike alternative theorems and mathematical programming, Optimization, vol. 16, pp. 643-650, 1985.

11. R.N. Kaul and S. Kaur, Optimality criteria in nonlinear programming involving nonconvex functions, J. Math. Anal. Appl., vol. 105, pp. 104-112, 1985. 
12. H. Kawasaki, Second-order necessary conditions of the Kuhn-Tucker type under new constraint qualifications, J. Optim. Theory Appl., vol. 57, no. 2, pp. 253-264, 1988.

13. S. Khurana, Symmetric duality in multiobjective programming involving generalized cone-invex functions, European J. Oper. Res., vol. 165, pp. 592-597, 2005.

14. S.K. Mishra and K.K. Lai, Second order symmetric duality in multiobjective programming involving generalized cone-invex functions, European J. Oper. Res., vol. 178, pp. 20-26, 2007.

15. M. Studniarski, Second-order necessary conditions for optimality in nonsmooth nonlinear programming, J. Math. Anal. Appl., vol. 154, pp. 303-317, 1991.

16. X.Q. Yang, On second-order directional derivatives, Nonlinear Analysis, Theory, Methods \& Applications, vol. 26, no. 1, pp. 55-66, 1996.

17. N.D. Yen and P.H. Sach, On locally Lipschitz vector valued invex functions, Bull. of Australian Math. Soc., vol. 47, pp. 259-272, 1993. 